\title{
DESEMPENHO PRELIMINAR DE CLONES DE SERINGUEIRA NA REGIÃO DE SÃO JOSÉ DO RIO PRETO, PLANALTO do ESTAdO DE SÃo PAULO $\left(^{1}\right.$ )
}

\author{
PAULO DE SOUZA GONÇALVES $\left({ }^{2,8}\right)$, MÁRIO CARDOSO $\left({ }^{3,8}\right)$, EVAIR MIESSE MENTE $\left(^{4}\right)$, \\ ANTONIO LÜCIO MELLO MARTINS $\left({ }^{5}\right)$, MARIA VITÓRIA CECCHETTI GOTTARDI $\left({ }^{6}\right)$ \\ e ALTINO ALDO ORTOLANI $\left({ }^{7,8}\right)$
}

\begin{abstract}
RESUMO
Neste trabalho, é apresentado o desempenho de nove anos de desenvolvimento e produção dos clones de seringueira [Hevea brasiliensis (Willd. ex. Adr. de Juss.) Muell.Arg.], a saber: AVROS 1328, PB 235, PB 217, GT 1, RRIM 701, IAN 873, Tab 821, IAC 15, Tab 804, IAC 222 e, como testemunha, RRIM 600. No experimento, instalado na Fazenda Água Milagrosa, município de Tabapuã, sob o delineamento de blocos casualizados com seis repetições, estudaram-se os seguintes caracteres: produção de borracha seca; vigor, expresso pela circunferência do caule; espessura de casca e árvores com seca do painel. Com relação à produção dos onze clones testados, PB 235, Tab 804, RRIM 701 e AVROS 1328 produziram, respectivamente, $113,45,38$ e $32 \%$ a mais em relação ao RRIM 600, em gramas/árvore/corte na média de quatro anos de sangria, onde, a partir do segundo ano, as plantas foram estimuladas. A maior parte desses clones se mostrou vigorosa, com caracteres secundários aceitáveis, tais como: espessura de casca virgem, árvores secas, baixa incidência de quebra pelo vento e baixa suscetibilidade de doenças de painel e folhas. O desempenho do GT 1 e do InC 222, embora vigoroso, não foi bom. RRIM 701 e IAC 15 apresentaram alta incidência de seca do painel; entretanto, o incremento da circunferência na sangria foi alto para esses clones.
\end{abstract}

Termos de indexação: seringueira, Hevea brasiliensis, desempenho de clones, vigor, produção.

(') Recebido para publicação em 28 de abril e aceito em 13 de outubro de 1993. Trabalho parcialmente financiado com recursos da FAPESP e do contrato IBAMA/EMBRAPA.

$\left({ }^{2}\right)$ Programa Seringueira da Divisão de Plantas Industriais (DPI), do Instituto Agronômico de Campinas (IAC), Caixa Postal 28, 13001-970 Campinas (SP).

$\left({ }^{3}\right)$ Divisão de Plantas Industriais (IAC). Aposentado.

( $\left.{ }^{4}\right)$ Gerente Agricola da Fazenda Água Milagrosa, Caixa Postal 23, 15880-000 Tabapuã (SP)

$\left({ }^{5}\right)$ Estą̧ão Experimental de Pindorama, IAC.

(6) Estagiária do Programa Seringueira (Bolsista da FAPESP).

( ${ }^{7}$ Seção de Climatologia Agrícola, IAC.

( ${ }^{8}$ ) Com bolsa de pesquisa do CNPq. 


\title{
ABSTRACT \\ PRELIMINARY PERFORMANCE OF CLONES OF RUBBER TREE \\ IN THE REGION OF SÃO JOSÉ DO RIO PRETO - PLATEAU OF THE STATE OF SÃO PAULO, BRAZIL
}

\begin{abstract}
The performance of growth and yield of nine years old rubber tree clones [Hevea brasiliensis (Willd. ex. Adr. de Juss.) Muell. Arg.], were evaluated. The studied clones were AVROS 1328, PB 235, PB 217, GT 1, RRIM 701, IAN 873, Tab 821, IAC 15, Tab 804, IAC 222 and RRIM 600 (check). The trial was established in the Água Milagrosa plantation in the region of São José do Rio Preto, under randomized complete blocks design with six replications. The studied characters were yield of dry rubber, vigor expressed by the stem girth, bark thickness and brown bast. From the second to fourth year of production the plants were stimulated. Among the tested clones PB 235, Tab 804, RRIM 701 and AVROS 1328 were outstanding in their performance out-yielding RRIM 600 by $113 \%, 45 \%, 38 \%$ and $32 \%$ respectively in the first four ycars of tapping. Most of these clones were vigorous with acceptable secondary characteristics such as virgin bark thickness, dry trees, low incidence of wind damage and low leaf and panel disease susceptibility. GT 1 and IAC 222 did not perform well in yield but was good in vigor. RRIM 701 and IAC 15 showed the highest brown bast incidence. However, girth increment on tapping was high for these clones.
\end{abstract}

Index terms: rubber tree, Hevea brasiliensis, clones performance, vigor, yield.

\section{INTRODUÇÃO}

No processo de melhoramento da seringueira [Hevea brasiliensis (Willd. ex. Adr. de Juss.) Muell. Arg.], a avaliação de clones constitui uma etapa que requer longo tempo atć a decisão final sobre aquele que melhor satisfaz aos critérios seletivos. Durante esse período de desenvolvimento, avaliaram-se os caracteres que determinam vigor e produção de borracha, além da incidência de doenças. Em geral, o próprio vigor do material tende a refletir sua adaptabilidade ao meio ambiente em que está scndo testado.

Tem sido freqüentemente relatado na avaliação de clones de seringueira que o desempenho é bem variável para diversos caracteres quantitativos sob as mais diferentes condições ambientais (Tan, 1980). Fatores como propriedades físicas e químicas do solo, temperatura e pluviosidade, afetam componentes do ciclo vegetativo dos clones avaliados (Pushparajah, 1983).

No Estado de São Paulo, onde a seringueira vem-se expandindo em áreas agroclimáticas bas- tante diferenciadas daquelas da região amazônica, o clima tem-se mostrado apropriado ao seu cultivo. Em muitos clones, as plantas alcançam circunferência do caule ideal à sangria em idade inferior a sete anos (Instituto Agronômico, 1989). Por outro lado, a grande variação dos elementos climáticos, como frio e déficit hídrico, observada nessas regiões ao longo dos anos de produção, possivelmente interfira nos processos fisiológicos das plantas. Em conseqüência, ocorrem variações no ciclo anual de produção de populações cstabelecidas em determinados locais (Ortolani, 1985).

Tendo em vista que a maioria das informações disponíveis sobre o comportamento dos clones recomendados para o plantio no Estado de São Paulo é extrapolada de outras regiões, ć de fundamental importância a avaliação do comportamento desses clones nas condições regionais. Com base nesses aspectos e objetivando selecionar os melhores clones para a região de São José do Rio Preto, onze clones foram postos em competição em um ensaio no município de Tabapuã (SP). 
O objetivo do presente trabalho foi identificar clones com alto potencial de produção, vigor e caracteres secundários satisfatórios para sua recomendação.

\section{MATERIAL E MÉTODOS}

O experimento foi instalado em janeiro de 1983, na Fazenda Água Milagrosa, no município de Tabapuã, em solo podzólico vermelho-amarelo de textura média, profundo, de topografia plana e bem drenado. Predomina nessa área o clima do tipo tropical continental, com características de verão úmido, níveis de encrgia típicos do trópico e um período de inverno seco com temperaturas e precipitações pluviais mais reduzidas. Em geral, o confronto entre as curvas mensais de evapotranspiração e da chuva resulta num equilíbrio favorável ao crescimento no período outubro-março e à produção de látex em novembro-julho. As deficiências hídricas $\mathrm{e}$ os baixos níveis térmicos ocorrem de junho ao início de setembro (Gonçalves ct al., 1992).

As mudas para o experimento foram preparadas pelo método convencional, onde as gemas maduras dos clones em estudo foram enxertadas em porta-enxertos de doze meses de idade originários de sementes de $H$. brasiliensis colhidas de uma população policlonal da Fazenda Água Milagrosa.

Em campo, o ensaio obedeceu ao delincamento de blocos ao acaso, com onze tratamentos, seis repetições e cinco plantas úteis por parccla no espaçamento de $7 \mathrm{~m}$ entre linhas de plantio e $3 \mathrm{~m}$ entre plantas.

O material que compõe o estudo é constituído de onze clones geneticamente superiores, de várias origens, descritos abaixo:

PB 235 e PB 217: Clones resultantes do cruzamento intra-específico de $H$. brasiliensis (PB 5/51 x PB 5/78) e (PB 5/51 x PB 6/9) respectivamente, ambos de origem malaia.

IAN 873: Clone amazônico obtido do cruzamento intra-específico de clones primários de $H$. brasiliensis originários da Malásia (PB 86) e do Brasil (FA 1717).

Tab 821 e Tab 804: Clones primários superiores de $H$. brasiliensis resultantes de seleções efetuadas em populações de genótipos da Fazenda Água Milagrosa.

RRIM 701: Clone terciário oriundo do cruzamento do clone primário 44/553 com o clone secundário RRIM 501 (Pil A 44 x Lun $\mathrm{N})$, ambos de origem malaia.

AVROS 1328: Clone secundário resultante dos clones primários AVROS 214 e AVROS 317 , ambos de $H$. brasiliensis selecionados em Sumatra, Indonésia.

GT 1: Clone primário de $H$. brasiliensis obtido de seleção efetuada na plantação Gondand Tapen, na Indonésia.

IAC 222: Clone poliplóide de $H$. brasiliensis oriundo da poliploidização do clone amazônico IAN 873.

IAC 15: Clone terciário intra-específico de $H$. brasiliensis, seleção do Instituto Agronômico, pelo cruzamento dos clones secundários RRIM 507 (Pil B $84 \times$ Pil A 44) com o RRIM 600 (Tjir 1 x PB 86), todos dc origem malaia.

RRIM 600: Clone secundário intraespecífico de $H$. brasiliensis, do cruzamento dos ćlones primários Tjir 1 com o PB 86, ambos de origem malaia.

Durante o período do desenvolvimento vegetativo dos clones, efetuaram-se mensurações anuais de circunferência do caule a $0,5 \mathrm{~m}$ acima do calo de enxertia no primeiro ano e a 1,2 $m$, começando do segundo ano. Dados de produção foram registrados a partir dos seis anos de idade para todas as plantas da parcela que apresentaram circunferência do caule superior a $45 \mathrm{~cm}$, a $1,2 \mathrm{~m}$ acima do calo de enxertia. No primciro ano, utilizou-se o sistema $S / 2 d / 2$. Do segundo ao quarto ano de sangria, as árvores foram submetidas ao sistema $\mathrm{S} / 2 \mathrm{~d} / 4 \mathrm{com}$ estimulação, utilizando-se o Ethrel a 2,5\% com 
duas aplicações a cada 45 dias no segundo ano e cinco aplicações a cada 45 dias no terceiro e no quarto ano. O registro da produção foi efetuado pelo látex coagulado nas tigelas ("biscoitos"), uma vez ao mês, seco em condições normais de sombra e ventilação por três meses e, então, pesado. A massa total mensal foi dividida pelo número de "biscoitos", sendo o resultado expresso em gramas/árvore/mês. A média das médias dos onze meses, considerando um período de descanso de reenfolhamento, foi expressa em gramas/árvore/ano, e a dos clones, em gramas/clone/ano. A partir desses dados, estimou-se a produção por clone/hectarc/ano, segundo recomendações da EMPRESA... (1989), adotando-se 140 cortes por ano, no sistema $\mathrm{S} / 2 \mathrm{~d} / 2$ e 60 cortes por ano no $\mathrm{S} / 2 \mathrm{~d} / 4$, sendo que no primeiro ano de sangria utilizaram-se, como cálculo, 240 plantas $(60 \%$ do estandc); no segundo, 340 (85\% do estande); no terceiro, 380 plantas (95\% do estande) e no quarto, 400 plantas (100\% do estande).

Amostras de casca para detcrminação da espessura de casca virgem foram tomadas a $1,0 \mathrm{~m}$ de altura do caule de cada árvorc, com o auxílio de um "vazador" e, em seguida, medidas com paquímetro.

Para a análise da variância, foram consideradas produções médias anuais de borracha seca, circunferências anuais do caule c espcssuras da casca aos seis anos de idade na abertura do painel e após o quarto ano de sangria.

Durante a execução do experimento, empregaram-se todas as práticas culturais convencionais ao cultivo da seringueira (Cardoso, 1982).

\section{RESULTADOS E DISCUSSÃo}

No quadro 1, são apresentados os valores dos quadrados médios obtidos mediante análise da variância para os quatro anos de vigor e de produção por árvore em gramas de borracha scca. Observam-se diferenças significativas a 0,01 de probabilidade para as referidas variáveis, indicando a existência de variabilidade para esses caracteres nessa população de clones.

\section{Vigor}

As médias de crescimento anual do caule, no que diz respcito aos períodos de imaturidade c adulto de cada clone, encontram-se no quadro 2. Esses dois periodos, relativos aos clones PB 235, GT 1, RRIM 701, Tab 804 e IAN 873 comparados à testemunha RRIM 600, são mostrados na figura 1; podemos observar que - clone PB 235 alcançou a média de $45 \mathrm{~cm}$ de circunferência do caule aos cinco anos, seguido dos clones IAN 873 e RRIM 701 com cinco anos $\mathrm{C}$ meio $\mathrm{C}$ seis anos respectivamente.

Quadro 1. Análise da variância para as variávcis circunferência do caule e produção de borracha seca, referentes a quatro anos de desenvolvimento do ensaio de clones de seringueira estabelecido na Fazenda Água Milagrosa, município de Tabapuã (SP)

\begin{tabular}{|c|c|c|c|c|c|c|c|c|c|}
\hline \multirow{2}{*}{ de $\begin{array}{l}\text { Fonte } \\
\text { variação }\end{array}$} & \multirow{2}{*}{ G1 } & \multicolumn{4}{|c|}{ Circunferência anual do caulc } & \multicolumn{4}{|c|}{ Produção anual de borracha seca } \\
\hline & & 1989 & 1990 & 1991 & 1992 & 1989 & 1990 & 1991 & 1992 \\
\hline & & 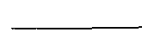 & $-c$ & & - & —. & 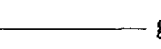 & $\mathrm{g}$ & 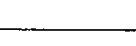 \\
\hline Bloco & 5 & $22,70^{\mathrm{ns}}$ & $23,00^{\mathrm{ns}}$ & $41,40^{\text {ns }}$ & $43,96^{\mathrm{ns}}$ & $16,07^{\text {ns }}$ & $152,92^{\mathrm{ns}}$ & $193,04^{\mathrm{ns}}$ & $55,05^{\mathrm{ns}}$ \\
\hline Clones & 10 & $155,35^{* *}$ & $194,37^{* *}$ & $245,29^{* *}$ & $350,65^{* *}$ & $74,86^{* *}$ & $1.286,76^{* *}$ & $1.496,62^{* *}$ & $309,30^{* *}$ \\
\hline Resíduo & 50 & 19,39 & 26,00 & 26,33 & 32,14 & 10,29 & 123,39 & 145,87 & 74,31 \\
\hline C.V.\% & & 9,89 & 10,52 & 10,01 & 10,22 & 21,19 & 31,98 & 25,38 & 20,24 \\
\hline Média geral & & 44,50 & 48,47 & 51,26 & 55,47 & 15,14 & 34,74 & 47,59 & 42,50 \\
\hline
\end{tabular}

* $\mathrm{P}>0,05 ; * * \mathrm{p}>0,01 ; \mathrm{ns}=$ não significativo. 
O clone RRIM 600 alcançou a média ideal de circunferência aos sete anos, enquanto o PB 217 mostrou o mais longo período de imaturidade, não alcançando, aos nove anos, a média de circunferência necessária para a abertura do painel. No período adulto, após a abertura do painel, o clone RRIM 701, que antes ocupava uma posição de destaque, foi superado pelo GT 1 no crescimento médio anual do caule. Esse fato pode ser explicado pelo atraso do seu crescimento após o procedimento de sangria.

O fotossintetizado, após a abertura do paincl, é repartido em duas fontes competidoras: látcx explotado e crescimento do caule. De acordo com Wycherley (1976), em alguns clones, sua maior parte é direcionada para o látex explotado, reduzindo o crescimento.

\section{Incremento do caule}

O quadro 3 mostra o incremento médio anual do crescimento do caule referente aos períodos de imaturidade e adulto dos nove anos de desenvolvimento vegetativo dos clones em estudo. A redução dos incrementos anuais, no tercciro, no quinto e no sexto ano do período de imaturidade se deve aos elevados totais anuais de deficiĉncia hídrica, da ordem de 364,231 e $362 \mathrm{~mm}$ respectivamente $\left({ }^{9}\right)$.

A maior média anual de crescimento no período de imaturidade foi observada no clone IAC $15,8,52 \mathrm{~cm}$, seguido pelos clones PB 235 , IAN 873 e Tab 804, com médias de $8,43,8,04$ e $7,94 \mathrm{~cm}$ respectivamente, todas superiores ao RRIM $600(6,94 \mathrm{~cm})$.

O maior incremento da circunferência, 16,68 $\mathrm{cm}$, foi apresentado pelo IAC 15, do tcrceiro para o quarto ano de desenvolvimento, seguido pelos clones Tab 804, IAN 873 e Tab 821, com valores de $15,60,15,46$ e $14,95 \mathrm{~cm}$ respectivamente (Quadro 3). Os três clones mais vigorosos (IAC 15, PB 235 e IAN 873.

( ${ }^{9}$ ) Arquivo de dados da Seção de Climatologia $\Lambda$ grícola - IAC, para Pindorama (SP).

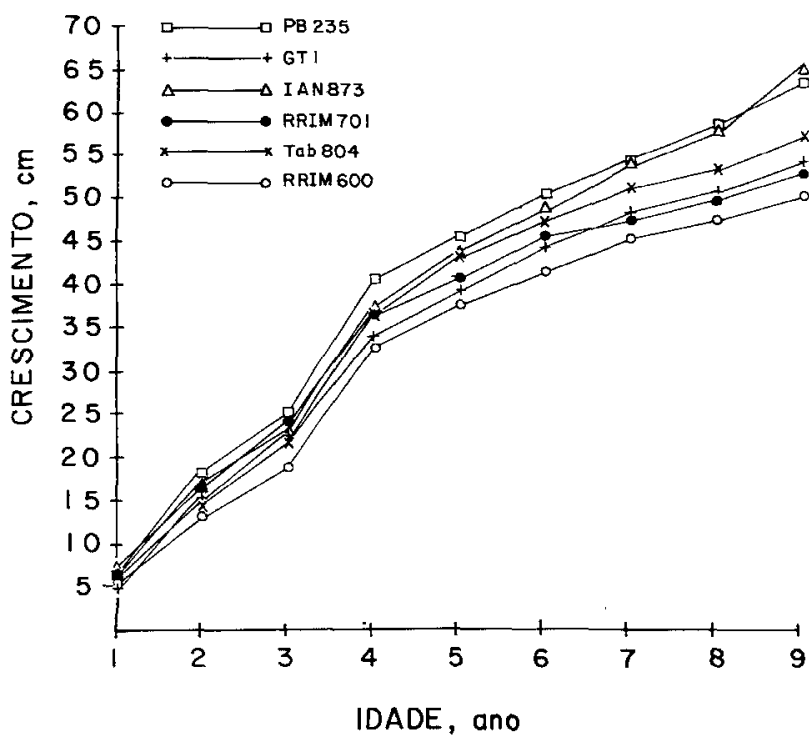

lïgura 1. Média de circunferência anual de seis clones selecionados.

Os três clones mais vigorosos (IAC 15, PB 235 e IAN 873) mostraram incremento anual de circunferência excedendo $8,0 \mathrm{~cm}$ por todo o período de imaturidade. A figura 2 mostra um modelo de " $\mathrm{V}$ " invertido de taxa de crescimento, cujo pico anual foi do terceiro ao quarto ano para a maioria dos clones.

O desenvolvimento dos clones no periodo adulto é um caráter muito importante para a manutenção da constância da produção, e que, por sua vez, contribuirá para a redução de quebra de árvores pelo vento.

Em geral, os incrementos são significativamente menores na maturidade (período adulto) comparado com o de imaturidade, indicando que o crescimento das árvores nesses clones sã́o afetados pela explotação do látex, discutido anteriormente.

A maior média anual de crescimento no pcríodo adulto foi do clone IAN 873, seguido dos clones Tab 821, IAC 15 e PB 235, com médias de $5,77,4,96,4,68$ e $4,32 \mathrm{~cm}$ respectivamente, todos bem superiores à média $(2,85$ cm) do RRIM 600. 
Quadro 2. Dados médios de circunferência do caule referentes a nove anos de desenvolvimento vegetativo de onze clones de seringueira estabelecidos em experimento em grande escala na Fazenda Água Milagrosa, no município de Tabapuã (SP)

\begin{tabular}{|c|c|c|c|c|c|c|c|}
\hline \multirow{3}{*}{ Clones } & \multirow{3}{*}{ Parentais } & \multicolumn{5}{|c|}{ Crescimento médio anual do caule } & \multirow{3}{*}{ Média } \\
\hline & & \multicolumn{5}{|c|}{ Pcriodo de imaturidade ${ }^{1}$ ) } & \\
\hline & & $1\left(\left(^{3}\right)\right.$ & $2\left(^{4}\right)$ & 3 & 4 & 5 & \\
\hline AVROS 1328 & AVROS $214 \times$ AVROS 317 & 6,79 & 14,46 & 19,92 & 33,50 & 37,26 & 22,39 \\
\hline PB 235 & $\mathrm{~PB} \quad 5 / 51 \quad \times \quad \mathrm{PB} \quad 5 / 87$ & 7,27 & 18,95 & 25,63 & 40,50 & 45,40 & 27,55 \\
\hline GT 1 & Clone Primário & 5,72 & 15,48 & 22,21 & 34,86 & 39,66 & 23,59 \\
\hline PB 217 & PB $5 / 51 \times$ PB $6 / 9$ & 5,35 & 11,12 & 14,23 & 26,32 & 31,05 & 17,61 \\
\hline RRIM 701 & $44 / 553 \times$ RRIM 501 & 7,38 & 17,65 & 24,27 & 36,47 & 40,30 & 25,21 \\
\hline IAN 873 & PB $86 \times$ B 1717 & 7,96 & 17,87 & 23,69 & 37,98 & 43,05 & 26,11 \\
\hline Tab 821 & Clone Primário & 8,06 & 16,13 & 22,48 & 37,43 & 41,40 & 25,10 \\
\hline IAC 15 & RRIM $507 \times$ RRIM 600 & 8,52 & 17,68 & 25,52 & 42,20 & 46,32 & 28,05 \\
\hline Tab 804 & Clone Primário & 7,01 & 15,74 & 22,36 & 37,97 & 43,00 & 25,22 \\
\hline IAC 222 & IAN 873 poliplóide & 8,38 & 16,74 & 23,15 & 32,16 & 35,41 & 23,17 \\
\hline RRIM 600 & Tjir $1 \times$ PB 86 & 6,58 & 13,85 & 19,03 & 33,32 & 37,91 & 22,14 \\
\hline Médias & & 7,18 & 15,97 & 22,04 & 35,70 & 40,07 & 24,19 \\
\hline
\end{tabular}

\begin{tabular}{|c|c|c|c|c|c|c|}
\hline \multirow{3}{*}{ Clones } & \multirow{3}{*}{ Parentais } & \multicolumn{4}{|c|}{ Crescimento médio anual do caule } & \multirow{3}{*}{ Média } \\
\hline & & \multicolumn{4}{|c|}{ Período adulto $\left(^{2}\right)$} & \\
\hline & & $6\left(^{5}\right)$ & 7 & 8 & 9 & \\
\hline & & & 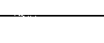 & $-\mathrm{cm}$ & 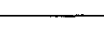 & 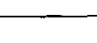 \\
\hline AVROS 1328 & AVROS $214 \times$ AVROS 317 & 41,25 & 43,83 & 46,00 & 50,04 & 45,28 \\
\hline PB 235 & $\mathrm{~PB} 5 / 51 \times \mathrm{PB} 5 / 87$ & 50,61 & 54,82 & 58,90 & 63,58 & 56,98 \\
\hline GT 1 & Clone Primário & 44,63 & 48,70 & 51,42 & 54,42 & 49,79 \\
\hline PB 217 & $\mathrm{~PB} 5 / 51 \times \mathrm{PB} 6 / 9$ & 34,32 & 37,78 & 39,90 & 42,81 & 38,70 \\
\hline RRIM 701 & $44 / 553 \times$ RRIM 501 & 45,14 & 48,56 & 50,17 & 53,71 & 49,39 \\
\hline IAN 873 & PB $86 \times$ B 1717 & 48,53 & 54,59 & 58,00 & 65,86 & 56,74 \\
\hline Tab 821 & Clone Primário & 45,38 & 49,40 & 54,67 & 60,25 & 52,42 \\
\hline IAC 15 & RRIM $507 \times$ RRIM 600 & 51,12 & 56,19 & 59,55 & 65,15 & 58,00 \\
\hline Tab 804 & Clone Primário & 47,68 & 51,10 & 53,37 & 57,11 & 52,31 \\
\hline IAC 222 & IAN 873 poliplóide & 39,23 & 42,56 & 44,25 & 47,13 & 43,29 \\
\hline RRIM 600 & Tjir $1 \times$ PB 86 & 41,62 & 45,60 & 47,65 & 50,17 & 46,26 \\
\hline Médias & & 44,50 & 48,47 & 51,26 & 55,47 & 49,92 \\
\hline
\end{tabular}

$\left({ }^{1}\right)$ O periodo de imaturidade é anterior à sangria. $\left({ }^{2}\right)$ o periodo adulto é posterior à sangria. $\left({ }^{3}\right)$ Dados coletados a $0,5 \mathrm{~m}$ acima do calo de enxertia. $\left({ }^{4}\right)$ A partir do segundo ano, os dados foram coletados a 1,2 m acima do cale de enxertia. $\left(^{5}\right.$ ) Abertura do painel. 
Quadro 3. Média do incremento anual do caule, referente a nove anos de crescimento vegetativo, do Experimento Avaliação de Clones em Grande Escala na Fazenda Água Milagrosa, no município de Tabapuã (SP)

\begin{tabular}{|c|c|c|c|c|c|c|c|c|}
\hline \multirow{3}{*}{ Clones } & \multirow{3}{*}{ Parentais } & \multicolumn{6}{|c|}{ Incremento médio anual do caule } & \multirow{3}{*}{ Média } \\
\hline & & \multicolumn{6}{|c|}{ Período de imaturidade $\left({ }^{1}\right)$} & \\
\hline & & $0-1$ & $1-2$ & $2-3$ & $3-4$ & $4-5$ & $5-6$ & \\
\hline & & \multicolumn{6}{|c|}{$\ldots$} & \\
\hline AVROS 1328 & AVROS $214 \times$ AVROS 317 & 6,79 & 7,67 & 5,45 & 14,37 & 3,77 & 3,45 & 6,92 \\
\hline PB 235 & $\mathrm{~PB} 5 / 51 \times \mathrm{PB} 5 / 87$ & 7,27 & 11,68 & 7,18 & 14,36 & 4,90 & 5,20 & 8,43 \\
\hline GT 1 & Clone Primário & 5,72 & 9,75 & 6,72 & 12,65 & 4,82 & 4,97 & 7,44 \\
\hline $\mathrm{PB} \quad 217$ & PB $5,51 \times P B \quad 6 / 9$ & 5,35 & 5,75 & 3,11 & 12,09 & 4,73 & 2,93 & 5,66 \\
\hline RRIM 701 & $44 / 53 \times$ RRIM 501 & 7,38 & 10,27 & 6,61 & 12,20 & 3,83 & 4,83 & 7,52 \\
\hline IAN 873 & $\mathrm{~PB} 86 \times \mathrm{B} 1717$ & 7,96 & 9,90 & 4,65 & 15,46 & 5,07 & 5,18 & 8,04 \\
\hline Tab 821 & Clone Primário & 8,06 & 8,06 & 6,35 & 14,95 & 3,97 & 3,98 & 7,56 \\
\hline IAC 15 & RRIM $507 \times$ RRIM 600 & 8,52 & 9,16 & 7,83 & 16,68 & 4,12 & 4,80 & 8,52 \\
\hline Tab 804 & Clone Primário & 7,01 & 8,72 & 6,62 & 15,60 & 5,03 & 4,68 & 7,94 \\
\hline IAC 222 & IAN 873 poliplóide & 8,38 & 8,34 & 6,41 & 9,01 & 3,25 & 3,82 & 6,53 \\
\hline RRIM 600 & Tjir $1 \times$ PB 86 & 6,58 & 7,27 & 5,17 & 14,29 & 4,60 & 3,72 & 6,94 \\
\hline Médias & & 7,18 & 8,78 & 6,01 & 13,79 & 4,37 & 4,32 & 7,41 \\
\hline
\end{tabular}

\begin{tabular}{|c|c|c|c|c|c|}
\hline \multirow{3}{*}{ Clones } & \multirow{3}{*}{ Parentais } & \multicolumn{3}{|c|}{ Incremento médio anual do caule } & \multirow{3}{*}{ Média } \\
\hline & & \multicolumn{3}{|c|}{ Período adulto $\left({ }^{2}\right)$} & \\
\hline & & $6-7$ & $7-8$ & $8-9$ & \\
\hline & & . & $\mathrm{m} / \mathrm{anc}$ & & \\
\hline AVROS 1328 & AVROS $214 \times$ AVROS 317 & 2,58 & 2,16 & 4,04 & 2,93 \\
\hline PB 235 & $\mathrm{~PB} 5 / 51 \times \mathrm{PB} 5 / 87$ & 4,21 & 4,07 & 4,69 & 4,32 \\
\hline GT 1 & Clone Primário & 4,07 & 2,71 & 3,00 & 3,26 \\
\hline PB 217 & $\mathrm{~PB} 5,51 \times \mathrm{PB} 6 / 9$ & 3,48 & 2,10 & 2,92 & 2,83 \\
\hline RRIM 701 & $44 / 53 \times$ RRIM 501 & 3,43 & 1,60 & 3,54 & 2,86 \\
\hline IAN 873 & PB $86 \times$ B 1717 & 6,06 & 3,40 & 7,86 & 5,77 \\
\hline Tab 821 & Clone Primário & 4,02 & 5,26 & 5,59 & 4,96 \\
\hline IAC 15 & RRIM $507 \times$ RRIM 600 & 5,08 & 3,36 & 5,60 & 4,68 \\
\hline Tab 804 & Clone Primário & 3,42 & 2,26 & 3,74 & 3,14 \\
\hline IAC 222 & IAN 873 poliplóide & 3,33 & 1,68 & 2,88 & 2,63 \\
\hline RRIM 600 & Tjir $1 \times$ PB 86 & 3,98 & 2,04 & 2,52 & 2,85 \\
\hline Médias & & 3,97 & 2,78 & 4,22 & 3,66 \\
\hline
\end{tabular}

( $\left.{ }^{1}\right)$ período de imaturidade é anterior à sangria. $\left({ }^{2}\right)$ O período adulto é posterior à sangria. 


\section{Abertura do painel}

A média de circunferência das plantas aptas à abertura dos painéis dos clones em estudo está relacionada com o percentual de plantas aptas à sangria (Quadro 4), o qual variou de $17 \%$ (PB 217) a $80 \%$ (IAC 15). Mais de $50 \%$ dos clones em estudo mostraram um percentual superior ao do RRIM 600 , com $43 \%$ de plantas aptas à sangria.

\section{Produção anuàl}

O quadro 5 apresenta a produção de borracha seca em gramas/árvore/corte, estimativas de produção de borracha seca por hectare/ano e percentagem de produção em relação à testemunha (RRIM 600). Considerando cerca de 240 árvores por hectare de plantas aptas à sangria, no primeiro ano, os clones PB 235, IAN 873 e Tab 821 foram os que mais produziram no primeiro ano de sangria, com produções de $22,76,16,96 \mathrm{c}$

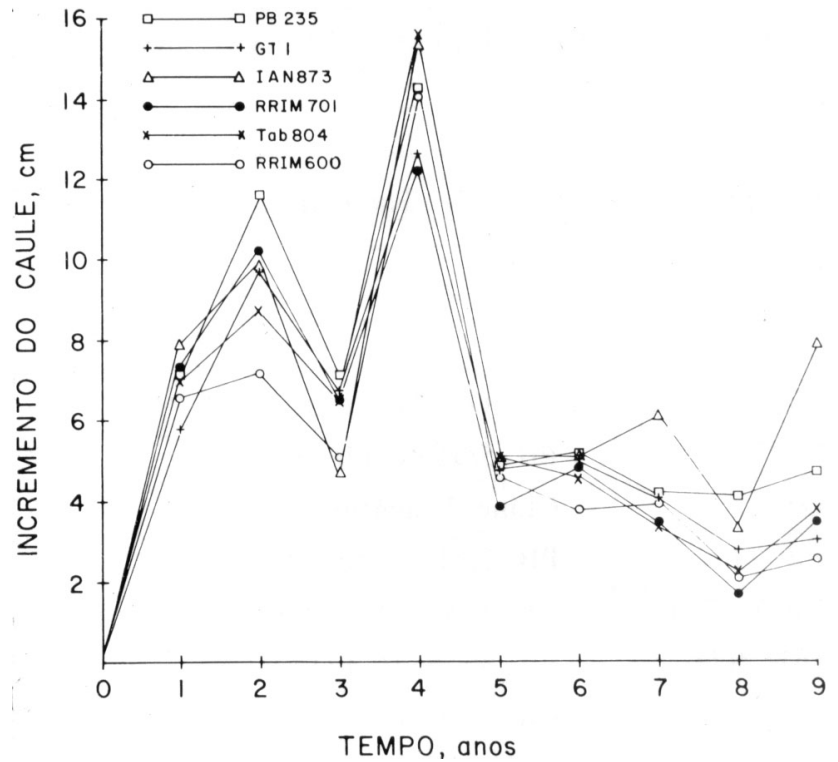

Figura 2. Média de incremento anual de circunferência do caule de seis clones selecionados.

Quadro 4. Classificação das características dos onze clones do Experimento Avaliação de Clones em Grande Escala na Fazenda Água Milagrosa, no município de Tabapuã (SP)

\begin{tabular}{|c|c|c|c|c|}
\hline Clone & $\begin{array}{l}\text { Circunferência média } \\
\text { do caule de } \\
\text { abertura do painel( }\left(^{1}\right)\end{array}$ & $\begin{array}{l}\text { Pcrcentual de } \\
\text { plantas aptas } \\
\text { à sangria }\end{array}$ & $\begin{array}{l}\text { Espessura de } \\
\text { casca virgem }\end{array}$ & $\begin{array}{l}\text { Árvores } \\
\text { secas }\left({ }^{2}\right)\end{array}$ \\
\hline & $\mathrm{cm}$ & $\%$ & $\mathrm{~mm}$ & $\%$ \\
\hline AVROS 1328 & $47,00 \pm 2,91\left(^{3}\right)$ & 43 & 7,93 & 5 \\
\hline PB 235 & $53,29 \pm 4,72$ & 67 & 7,44 & 20 \\
\hline GT 1 & $49,15 \pm 3,93$ & 43 & 6,45 & 15 \\
\hline PB 217 & $48,60 \pm 4,04$ & 17 & 6,41 & 10 \\
\hline RRIM 701 & $49,70 \pm 2,76$ & 57 & 7,17 & 25 \\
\hline IAN 873 & $51,59 \pm 5,59$ & 73 & 6,53 & 0 \\
\hline Tab 821 & $49,70 \pm 3,68$ & 57 & 7,20 & 16 \\
\hline IAC 15 & $51,42 \pm 4,22$ & 80 & 7,63 & 25 \\
\hline Tab 804 & $49,95 \pm 4,86$ & 63 & 6,40 & 10 \\
\hline IAC 222 & $46,33 \pm 2,60$ & 30 & 7,19 & 8 \\
\hline RRIM 600 & $47,61 \pm 2,81$ & 43 & 6,56 & 5 \\
\hline Médias & $49,48 \pm 3,83$ & 52 & 6,99 & - \\
\hline
\end{tabular}

${ }_{\left({ }_{3}\right)}^{1}$ Árvores com circunferência superior a $45 \mathrm{~cm}$ a $1,20 \mathrm{~m}$ de altura. $\left({ }^{2}\right)$ Foram incluídas também árvores com tendência a secamento.

(3) Desvio-padrão da média. 
$16,01 \mathrm{~g}$ de borracha seca/árvore/corte respectivamente, todos superiores ao RRIM 600 $(12,44 \mathrm{~g})$, considerado um dos mais plantados no planalto paulista.

No segundo ano, com uso de estimulante, houve um aumento significativo de produção para a maioria dos clones (Quadro 5).

O PB 235, bastante superior aos outros clones, com produção de $74,99 \mathrm{~g}$, seguido dos clones Tab 804, AVROS 1328 e RRIM 701, com produções de $39,94,38,63$ e 38,35 g respectivamente, todos superiores ao RRIM 600 $(24,30 \mathrm{~g})$. Comparando com os dados da produção estimulada da Malásia (Gonçalves et al., 1991), o clone RRIM 600 não correspondeu à expectativa de produção, levando a crer que o referido genótipo não respondeu à dosagem de estimulante aplicada. Exceto o clone IAC 222, com 22,98 g, os demais apresentaram produções superiores ao RRIM 600 .

No terceiro ano, também com estimulação, houve um aumento significativo da produção, fazendo acreditar que a freqüência de cinco aplicações a cada 45 dias contribuiu mais ainda para o aumento de produção. Seguidos do PB 235 , com produção de $85,92 \mathrm{~g}$, os clones Tab 804, RRIM 701 e AVROS 1328 apresentaram produções de $61,17,56,21$ e $47,63 \mathrm{~g}$ de borracha seca/árvore/sangria respectivamente, todos superiores ao clone RRIM 600 , com $34,63 \mathrm{~g}$.

Exceto para os clones IAC 222, AVROS 1328, RRIM 600, IAC 15 e IAN 873, houve redução de produção no quarto ano de sangria para a maioria dos clones estudados. Uma das hipóteses para essa queda acentuada é atribuída à incidência de ácaro ocorrida no período, causador do amarelecimento e da queda das folhas.

A produção de borracha seca dos quatro anos relativos aos clones PB 235, GT 1, RRIM 701, Tab 804 e IAN 873, em relação à testemunha, RRIM 600 , é mostrada na figura. 3 .

\section{Produção acumulada}

A produção acumulada dos primeiros quatro anos de sangria (Quadro 5) foi maior para o

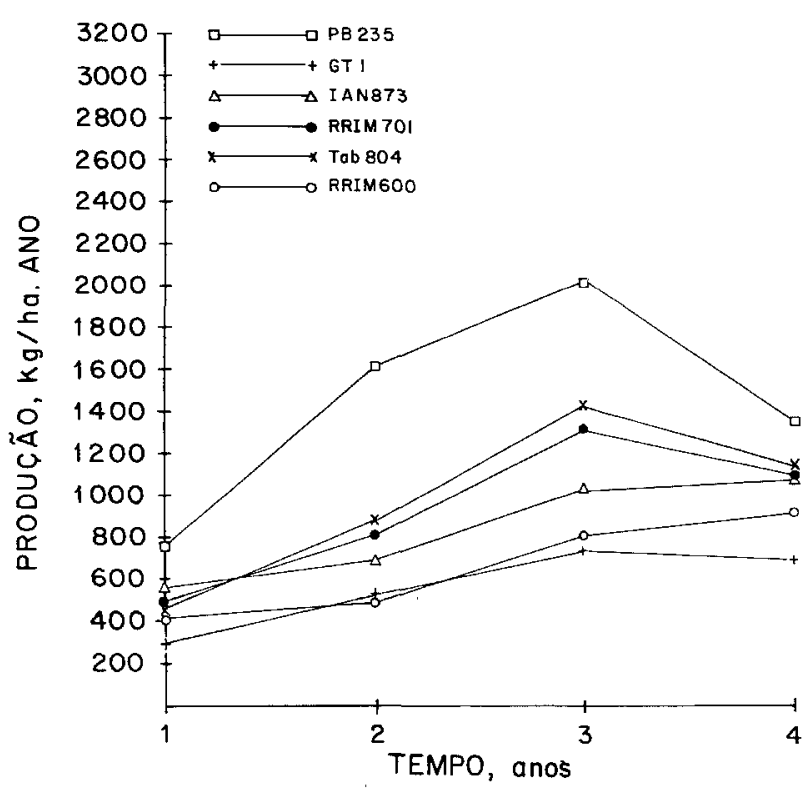

Figura 3. Produção média anual comparativa de seis clones selecionados.

PB 235, com $5.612 \mathrm{~kg} / \mathrm{ha} / \mathrm{ano}$ de borracha seca, superior à testemunha, RRIM 600 , em $113 \%$, seguida dos clones Tab 804 e RRIM 701, que produziram 45 e $38 \%$ a mais que o RRIM 600 respectivamente. O GT 1 foi o único com produção acumulada inferior ao RRIM 600 .

Por ser tido como um dos clones mais plantados no Estado, causa preocupação observar que o Gt 1, nos primeiros quatro anos de produção, mostra resultados desanimadores, abaixo do RRIM 600. Resultados semelhantes foram observados por Chan (1989) na Malásia. Possuidor de vários atributos, a maior desvantagem do GT 1, é sua baixa produção nos primeiros anos, embora seu vigor no período de imaturidade seja bem semelhante ao do RRIM 600. É interessante observar a comparação do desempenho de ambos, quando os pré-requisitos para alta produtividade precoce são: bom vigor e alta produção nos primeiros anos, caracteres esses que colocam o PB 235 e o Tab 804 no topo da lista de clones de bom desempenho. O desempenho do PB 217 e do IAC 222 também foi desanimador. Resta-nos dar continuidade à avaliação no que diz respeito a painéis 
de casca regenerada e outros caracteres secundários. Existem casos na literatura (Chan, 1989) de clones que declinaram sua produção no painel regenerado, em diferentes ambientes.

\section{Espessura de casca}

A espessura de casca virgem aos seis anos de idade - Quadro 4 - mostrou valores que variaram de $6,40 \mathrm{~mm}$ (Tab 804) a $7,93 \mathrm{~mm}$
(AVROS 1328), destacando-se ainda os clones IAC 15, PB 235 e Tab 821, com médias de $7,63,7,44$ e $7,20 \mathrm{~mm}$ respectivamente, todos superiores ao RRIM 600, cuja média foi de $6,56 \mathrm{~mm}$. É importante enfatizar que uma casca espessa, segundo Simmonds (1989), é que, além do maior número de anéis de vasos laticíferos, proporciona maior facilidade no procedimento da sangria.

Quadro 5. Estimativa de produção de borracha seca e percentagem em relação à testemunha de dez clones estabelecidos em experimento em grande escala na Fazenda Água Milagrosa, no município de Tabapuã (SP)

\begin{tabular}{|c|c|c|c|c|c|c|c|c|}
\hline \multirow{2}{*}{ Clones( $\left.{ }^{1}\right)$} & \multirow{2}{*}{ Parentais } & \multicolumn{4}{|c|}{ Produção } & \multicolumn{2}{|c|}{ Produção - 4 anos } & \multirow{2}{*}{$\begin{array}{l}\text { Porcentagem } \\
\text { em relação } \\
\text { à testemunha }\end{array}$} \\
\hline & & $1\left(^{2}\right)$ & $2\left(^{3}\right)$ & $3\left(^{4}\right)$ & $4\left(^{5}\right)$ & Acumulada & Médias & \\
\hline & & 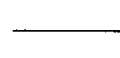 & $-\mathrm{kg} / \mathrm{ha} / \mathbf{a}$ & $g / c / a\left({ }^{6}\right)$ & & & & $\%$ \\
\hline \multirow[t]{2}{*}{ AVROS 1328} & AVROS $214 \times$ AVROS 317 & 457 & 788 & 1086 & 1154 & 3485 & 871 & 132 \\
\hline & & $(13,59)$ & $(38,63)$ & $(47,63)$ & $(48,07)$ & - & $(36,98)$ & 134 \\
\hline \multirow[t]{2}{*}{ PB 235} & $\mathrm{~PB} 5 / 51 \times \mathrm{PB} 5 / 87$ & 765 & 1530 & 1959 & 1361 & 5615 & 1404 & 213 \\
\hline & & $(22,76)$ & $(74,99)$ & $(85,92)$ & $(56,70)$ & - & $(60,09)$ & 218 \\
\hline \multirow[t]{2}{*}{ GT 1} & Clone primário & 300 & 499 & 715 & 700 & 2214 & 553 & 84 \\
\hline & & $(8,95)$ & $(24,46)$ & $(31,36)$ & $(29,19)$ & - & $(23,49)$ & 85 \\
\hline \multirow[t]{2}{*}{ PB 217} & $\mathrm{~PB} 5 / 51 \times \mathrm{PB} \quad 6 / 9$ & 624 & 635 & 1037 & 940 & 3236 & 809 & 122 \\
\hline & & $(18,57)$ & $(31,14)$ & $(45,49)$ & $(39,17)$ & - & $(33,59)$ & 122 \\
\hline \multirow[t]{2}{*}{ RRIM 701} & 44/553 X RRIM 501 & 500 & & 1281 & 1083 & 3646 & 911 & 138 \\
\hline & & $(14,87)$ & $(38,35)$ & $(56,21)$ & $(45,14)$ & - & $(38,64)$ & 140 \\
\hline \multirow[t]{2}{*}{ IAN 873} & PB $86 \times$ B 1717 & 570 & 655 & 1007 & 1081 & 3313 & 828 & 125 \\
\hline & & $(16,96)$ & $(32,09)$ & $(44,17)$ & $(45,06)$ & - & $(34,57)$ & 125 \\
\hline \multirow[t]{2}{*}{ Tab 821} & Clone primário & 538 & 590 & 1058 & 988 & 3174 & 793 & 120 \\
\hline & & $(16,01)$ & $(28,93)$ & $(46,40)$ & $(41,17)$ & - & $(33,13)$ & 120 \\
\hline \multirow[t]{2}{*}{ IAC 15} & RRIM $507 \times$ RRIM 600 & 460 & 543 & 859 & 873 & 2735 & 684 & 104 \\
\hline & & $(13,67)$ & $(26,62)$ & $(37,66)$ & $(36,39)$ & - & $(28,58)$ & 103 \\
\hline \multirow[t]{2}{*}{ Tab 804} & Clone primário & 484 & 815 & 1395 & 1145 & 3839 & 960 & 145 \\
\hline & & $(14,40)$ & $(39,94)$ & $(61,17)$ & $(47,70)$ & - & $(40,80)$ & 148 \\
\hline \multirow[t]{2}{*}{ IAC 222} & IAN 973 poliplóide & 480 & 469 & 739 & 979 & 2667 & 667 & 101 \\
\hline & & $(14,26)$ & $(22,98)$ & $(32,42)$ & $(40,79)$ & - & $(27,61)$ & 100 \\
\hline \multirow[t]{2}{*}{ RRIM 600} & Tjir $1 \times$ PB 86 & 418 & 496 & 789 & 936 & 2639 & 660 & 100 \\
\hline & & $(12,44)$ & $(24,30)$ & $(34,63)$ & $(39,00)$ & - & $(27,59)$ & 100 \\
\hline
\end{tabular}

$\left.{ }^{1}\right)$ Idade de abertura do painel: 6 anos. $\left({ }^{2}\right)$ Sangria no sistema $S / 2$ d/2: considerou-se um estande de 240 árvores (60\%) e 140 cortes/ano. $\left({ }^{3}\right)$ Sangria no sistema S/2 d/4 com estimulação, ET 2,5\%: considerou-se um estande de 340 árvores $(85 \%)$ e 60 cortes/ano. (4) Sangria no sistema $S / 2 \mathrm{~d} / 4$ com estimulação, ET 2,5\%: considerou-se um estande de 380 árvores $(95 \%)$ e 60 cortes/ano. ( $\left.{ }^{5}\right)$ Sangria no sistema $\mathrm{S} / 2 \mathrm{~d} / 4 \mathrm{com}$ estimulação, ET $2,5 \%$ : considerou-se um estande de 400 árvores $(100 \%)$ e 60 cortes/ano. ( $\left.{ }^{6}\right) \mathrm{kg} / \mathrm{ha} / \mathrm{a}$ : quilograma/hectare/ano e g/c/a: gramas/corte/árvore (valores entre parênteses). 


\section{Seca do painel}

A incidência de árvores secas - Quadro 4 - mostrou maior percentual para os clones RRIM 701 e IAC 15, ambos com $25 \%$, seguidos do PB 235, Tab 821 e GT 1 com 20, 16 e $15 \%$ respectivamente, com maior incremento de árvores a partir do terceiro ano de sangria. Entretanto, o número de árvores secas para os outros clones foi considerado moderado quando comparado com os referidos clones. É interessante enfatizar que a seca do painel é uma enfermidade fisiológica de seringueiras em regime de sangria e que aumenta em alguns clones com a intensidade da explotação (Sharples \& Lambourne, 1924, Bealing \& Chua, 1972). É um caráter inerente ao clone influenciado pela intensidade de sangria e pela estimulação.

\section{Suscetibilidade a pragas e doenças}

Em geral, não houve grande incidência de clones com doenças de folhas, exceto de ácaro, no terceiro e no quarto ano.

Foi observada baixa incidência de antracnose do painel para todos os clones.

\section{CONCLUSÕES}

As conclusões do presente trabalho são ainda preliminares e se baseiam em produção, vigor e outros caracteres secundários dos primeiros quatro anos de sangria, em resumo, podendo ser tiradas as seguintes inferências:

1. Os clones PB 235, IAN 873, Tab 804 e RRIM 701 apresentaram o melhor desempenho no decorrer do período de avaliação.

2. Os clones PB 217 e Tab 821 revelaram resultados intermediários e pouco produtivos no quadriênio. Os clones IAC 15 e IAC 22 foram inferiores em produção, próximos da testemunha.

3. O GT 1, um dos clones mais universais, muito plantado no planalto paulista, mostrou produção muito baixa e inferior à do RRIM 600 nos quatro primeiros anos.

\section{AGRADECIMENTOS}

Os autores agradecem ao Dr. Alberto Ortemblad, proprietário da Fazenda Água Milagrosa, a colaboração na instalação, condução e coleta de informações durante os levantamentos; ao Técnico Agropecuário Edison Bernardo de Freitas, da Estação Experimental de Pindorama, o desempenho em algumas coletas de informações.

\section{REFERÊNCIAS BIBLIOGRÁFICAS}

BEALING, F.J. \& CHUA, S.E. Output, composition and metabolic activity of Hevea latex in relation to tapping intensity and the onset of brown bast. Journal of the Rubber Research Institute of Malaya, Kuala Lumpur, 23(3):204-231, 1972.

CARDOSO, M. Instruções para a cultura da seringueira. 2.ed. Campinas, Instituto Agronômico, 1980. 42p. (Boletim, 196)

CHAN, W.H. Commercial performance of clones planted in the last fifteen years in a large group of rubber states. In: RUBBER GROWERS CONFERENCE, Kuala Lumpur, 1989. Proceedings. Kuala Lumpur, Rubber Research Institute of Malaysia, 1989. p.36--52.

EMPRESA BRASILEIRA DE PESQUISA AGROPECUÁRIA. Centro Nacional de Pesquisa de Seringueira e Dendê. Melhoramento genético da seringueira. Manaus, 1989. 23p. (Documentos, 10)

GONÇALVES, P. de S.; CARDOSO, M. \& SAES, L.A. Desempenho de quatro clones de seringueira na região do Vale do Ribeira, SP. Pesquisa Agropecuária Brasileira, Brasilia, 26(5):681-690, 1991.

GONÇALVES, P. de S.; GORGULHO, E.P.; MARTINS, A.L.M.; BORTOLETTO, N.; CARDOSO, M. \& BERMOND, G. Variação genética de componentes do crescimento em progênies jovens de uma população de clones de seringueira. Bragantia, Campinas, 51(2): 161-171, 1992.

INSTITUTO AGRONÔMICO (Campinas). Programa Seringueira: relatório anual 1989. Campinas, 1989. n.p. (Datilografado)

ORTOLANI, A.A. Aptidão climática para cultura da seringueira em Minas Gerais. Informe Agropecuário, Belo Horizonte, 11(121):8-12, 1985. 
PUSHPARAJAH, E. Problems and potentials for establishing Hevea under difficult environmental conditions. Planter, Kuala Lumpur, 50:242-251, 1983.

SHARPLES, A. \& LAMBOURNE, J. Field experiments relating to brown bast disease of Hevea brasiliensis. Malayan Agricultural Journal, Kuala Lumpur, 12:290-296, 1924.

SIMMONDS, N.W. Rubber breeding. In: WEBSTER, C.C. \& BAULKWILL, W.J., eds. Rubber. London, Longman, 1989. Cap. 3, p. 85-124.
TAN, H. Estimates of genetic parameters and their implications in Hevea breeding. In: INTERNATIONAL CONGRESS SABRAO, 4., Kuala Lumpur, 1981. Crop improvement research: proceedings. Kuala Lumpur, Society for Advancement of Breeding Researches in Asia and Oceania, 1981. p.439-446.

WYCHERLEY, P.R. Tapping and partition. Journal of the Rubber Research Institute of Malaysia, Kuala Lumpur, 24:169-194, 1976. 